\title{
Heterogeneity of Vaccine-Induced Immune Thrombotic Thrombocytopenia after ChAdOx1 nCoV-19 Vaccination and Safety of Second Vaccination with BNT162b2
}

\author{
Edelgard Lindhoff-Last ${ }^{1}$ Linda Schoenborn ${ }^{2}$ Michael Piorkowski ${ }^{1}$ Joerg Herold ${ }^{3}$ \\ Andreas Greinacher $^{2}$ Jo-Ann Sheppard ${ }^{4}$ Theodore E. Warkentin ${ }^{4}$
}

${ }^{1}$ Cardioangiology Center Bethanien Hospital, CCB Coagulation Center and CCB Coagulation Research Center, Frankfurt, Hessen, Germany

${ }^{2}$ Department of Immunology and Transfusion Medicine, University Hospital Greifswald, Greifswald, Germany

${ }^{3}$ Department of Vascular Medicine/Angiology, Städtisches Klinikum Darmstadt, Darmstadt, Germany

${ }^{4}$ Department of Pathology and Molecular Medicine, McMaster

University, Hamilton, Ontario, Canada

Thromb Haemost 2022;122:304-307.

Vaccine-induced immune thrombotic thrombocytopenia (VITT) is a rare side effect of two adenoviral vector vaccines, ChAdOx1 nCoV-19 (AstraZeneca) and Ad26.CoV2.S (Johnson \& Johnson/Janssen); it is caused by platelet-activating immunoglobulin $\mathrm{G}(\mathrm{IgG})$ that recognizes platelet factor 4 (PF4), as shown by positive testing by PF4/heparin-enzyme-linked immunosorbent assay (PF4-H-ELISA) in combination with PF4-enhanced washed platelet activation assays (PF4PAA). ${ }^{1-3}$ Clinical presentation is heterogeneous, ${ }^{4}$ with some patients presenting without overt thrombosis (VITT with isolated thrombocytopenia) or with severe headache. ${ }^{3,5,6}$ Few data exist regarding long-term decline in PF4-dependent antibodies ${ }^{7}$; in addition, there is uncertainty about timing and safety of subsequent booster vaccination after an episode of VITT.

We report four cases of VITT ( 3 females, aged 38, 56, and 76 years; and 1 male, aged 32 years) that illustrate its diverse clinical spectrum (see - Fig. 1A-D for details). Two patients (patients 1 and 2) had thrombocytopenia associated with both arterial and venous thromboses, while one (patient 3 ) had lower limb venous thrombosis without thrombocytopenia. The most unusual case was patient 4 , who had thrombocytopenia together with severe, persistent headache and abdominal pain/transaminitis; however, imaging studies

received

October 3, 2021

accepted

November 11, 2021

published online

November 18, 2021
Address for correspondence Edelgard Lindhoff-Last, MD, Cardioangiology Center Bethanien Hospital, CCB Coagulation Center and CCB Coagulation Research Center, Im Prüfling 23, Frankfurt, Hessen 60389, Germany (e-mail: e.lindhoff-last@ccb.de).

were negative for cerebral and abdominal thromboses, and symptoms resolved in association with early anticoagulation therapy.

\section{Results of PF4-Dependent Antibodies}

For detection of PF4-dependent antibodies, a PF4-H ELISA (in house assay of the Greifswald Laboratory ${ }^{1,8}$ ) and a chemiluminescent immunoassay for detection of heparin-induced thrombocytopenia (HIT) antibodies (CLIA, Werfen) were performed. In addition, heparin-induced platelet activation assay (HIPA) and PF4-PAA were analyzed in available blood samples. ${ }^{9}$ In all patients, PF4-dependent antibodies were detected. There was heterogeneity in reaction profiles: three patients presented with PF4-H-ELISA antibodies while two patients (including the ELISA-negative patient) had positive results in the PF4-PAA. The HIPA and the chemiluminescent immunoassay for detection of HIT antibodies (CLIA assay) remained negative in all patients. During follow-up, results of PF4-H-ELISA became negative in only one of these patients after 3 months, while the PF4-H-ELISA antibodies persisted for more than 5 months in the other two patients. PF4-PAA became negative (at 1- and 3-month follow-up) in both patients with initially positive results.

\section{(c) 2021. The Author(s).}

This is an open access article published by Thieme under the terms of the Creative Commons Attribution-NonDerivative-NonCommercial-License, permitting copying and reproduction so long as the original work is given appropriate credit. Contents may not be used for commercial purposes, or adapted, remixed, transformed or built upon. (https://creativecommons.org/ licenses/by-nc-nd/4.0/) Georg Thieme Verlag KG, Rüdigerstraße 14, 70469 Stuttgart, Germany 

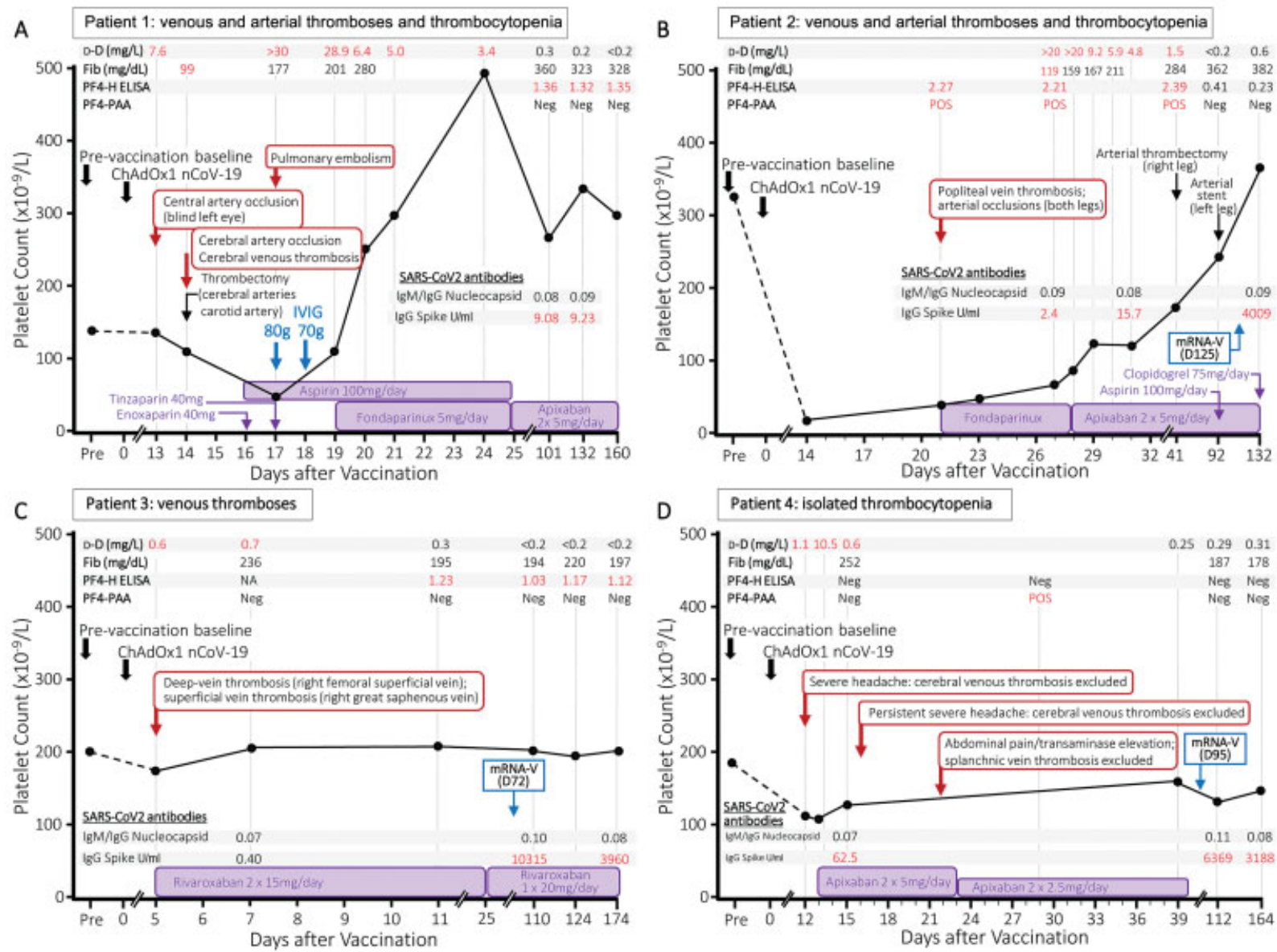

Fig. 1 (A-D) Clinical course of four patients with heterogenous presentations of vaccine-induced immune thrombotic thrombocytopenia (VITT). "Day 0" indicates the date of first vaccination with ChAdOx1nCoV-19 (AstraZeneca). The inset shows results of D-dimer (d-D; normal range $<0.5 \mathrm{mg} / \mathrm{L}$ ), fibrinogen (Fib; normal range $<1.5 \mathrm{mg} / \mathrm{dL}$ ), and testing for VITT antibodies. In all patients, four assays for VITT/HIT antibodies were performed: a PF4/heparin enzyme-linked immunosorbent assay (PF4-H ELISA), a chemiluminescent immunoassay for detection of HIT antibodies (CLIA, Werfen), a heparin-induced platelet activation assay (HIPA), and a PF4-enhanced washed platelet activation assay (PF4-PAA). Since none of the patients developed positive results in the HIPA-assay and the CLIA-assay, these negative results are not shown in the figure. (A) Patient 1 (female, 38 years) presented with thrombocytopenia associated with arterial and venous thromboses and the platelet count increased rapidly after two doses of IVIG. Persistent PF4-H ELISA positivity was observed. The patient declined repeat vaccination. (B) Patient 2 (female, 76 years) had severe thrombocytopenia and arterial and venous thromboses. Clinical symptoms improved during anticoagulation, without IVIG application. PF4-H ELISA and PF4-PAA declined over time. Second vaccination with BNT162b2 (day 125, Pfizer-BioNTech) was successfully applied under oral anticoagulation after VITT antibodies had become negative and vaccination was well tolerated. (C) Patient 3 (male, 32 years) presented with isolated venous thrombosis without thrombocytopenia. PF4-H ELISA antibodies persisted and successful vaccination with BNT162b2 (day 72, Pfizer-BioNTech) was performed without side effects under oral anticoagulation. (D) Patient 4 (female, 56 years) had thrombocytopenia with high D-dimer levels but venous thromboses were excluded despite severe headache and abdominal pain. She received early oral anticoagulation until day 51 after vaccination. The PF4-PAA was once positive and became quickly negative. Second vaccination with BNT162b2 (day 95, Pfizer-BioNTech) was successfully applied after stop of anticoagulation. IVIG, intravenous immunoglobulin; PF4, platelet factor 4.

\section{Results of Thrombophilia Screening}

Inherited and acquired thrombophilia including factor $\mathrm{V}$ Leiden mutation, prothrombin mutation, protein C-, protein S-, and antithrombin-deficiency, antiphospholipid antibodies, and HIT was excluded in all patients with thrombosis.

\section{Clinical Course and Treatment of Patients}

Patient 1 (see - Fig. 1A) presented with severe headache and elevated D-dimer on day 13 after vaccination. Cerebral computed tomography (CT) angiography on admission showed no vascular occlusions, thrombosis, or fresh ische- mia. Immediately following CT, there was visual disturbance in the left eye, nausea, and vomiting. Lysis therapy was initiated. One day later, after starting anticoagulation with low-molecular-weight heparin (LMWH), the platelet count dropped to $40 \times 10^{9} / \mathrm{L}$ and the patient developed right hemisymptomatics with motor aphasia. Subsequent CT angiography revealed a new occlusion of the middle cerebral artery and cerebral vein thrombosis. Arterial thrombectomy was performed successfully and neurological clinical symptoms improved. LMWH was stopped, intravenous immunoglobulins were given twice (day 17 and day 18) and aspirin was initiated, followed by additional fondaparinux ( $5 \mathrm{mg}$ once daily) after an increase in platelet count. On day $17, \mathrm{CT}$ thorax 
revealed asymptomatic central pulmonary artery embolism as incidental finding. After initiation of fondaparinux therapy, there was a further increase in platelet count, aspirin was stopped, and the patient was switched to long-term oral anticoagulation with therapeutic dose apixaban $5 \mathrm{mg}$ twice daily.

Patient 2 (see - Fig. 1B) developed diarrhea from day 8 after vaccination. From day 13 extensive hematomas developed in both legs. In addition, walking distance was acutely reduced to $10 \mathrm{~m}$ and a drop of platelet count to $20 \times 10^{9} / \mathrm{L}$ was diagnosed by the family doctor. After admission to hospital, popliteal vein thrombosis and arterial occlusions in both legs were diagnosed on day 21. Initially low-dose fondaparinux $2.5 \mathrm{mg}$ once daily was started due to the hematomas and low platelet count. After increase of platelet count, the patient was switched to apixaban $5 \mathrm{mg}$ twice daily (day 27). In the further course, arterial thrombectomy and stenting were performed in both legs followed by additional platelet aggregation inhibition.

Patient 3 (see - Fig. 1C) presented with low D-dimer elevation and venous thrombosis of the right leg on day 5 after vaccination. Apart from vaccination no other risk factors for venous thrombosis were identified. Rivaroxaban $15 \mathrm{mg}$ twice daily was started followed by rivaroxaban $20 \mathrm{mg}$ once daily 3 weeks after initiation of oral anticoagulation.

Patient 4 (see - Fig. 1D) presented with severe headache, a drop in platelet count, and elevated D-dimer on day 12 after vaccination. Within 1 day after first presentation, D-dimer increased to $10.5 \mathrm{mg} / \mathrm{L}$ and therapeutic oral anticoagulation with apixaban $5 \mathrm{mg}$ twice daily was immediately started because a "pre-VITT" syndrome was suspected by the treating physician. Platelet count increased and cerebral vein thrombosis was successfully excluded twice. On day 23 abdominal pain and transaminase elevation was observed but splanchnic vein thrombosis was excluded by abdominal magnetic resonance venography.

\section{Second Vaccination with BNT162b2}

Second vaccination with the messenger RNA (mRNA) vaccine, BNT162b2 (Pfizer-BioNTech) was performed in three of the patients, without thrombocytopenia or other adverse sequelae (the fourth patient declined repeat vaccination); for the patient (patient 3) whose PF-H-ELISA remained positive at the time of booster vaccination, no increase in subsequent ELISA reactivity was seen. After the second vaccination with the mRNA-vaccine, severe acute respiratory syndrome coronavirus 2 (SARS-CoV2) IgG Spike antibodies increased in the three second-vaccinated patients while SARS-CoV2 antibodies against IgM/IgG nucleocapsid antibodies remained negative (for details, see $\boldsymbol{-}$ Fig. 1B-D).

\section{Limitations}

Patient 1 presented with clinical symptoms of VITT in March 2021. At that time the syndrome was relatively unknown and specific laboratory assays for diagnosis of PF4-induced antibodies were not yet available. High-dose immunoglobulins were applied and the PF4-PAA assay was first measured 3 months after VITT. This assay was negative while the PF4-H ELISA was persistently positive. The application of immunoglobulins might have caused the negative test result in the PF4-PAA assay. In addition, it is known that PF4-PAA assay results decline earlier than the PF4-ELISA assay results. ${ }^{7}$

Patient 3 presented with venous thrombosis without thrombocytopenia. PF4 H-ELISA assays remained positive while the PF4-PAA assay was negative. Although up to $6.8 \%$ of patients vaccinated with ChAdOx1 nCov-19 or BNT162b2 may develop low-titer PF4-H-ELISA antibodies without clinical relevance, ${ }^{9}$ patient 3 developed high-titer PF4-H-ELISA antibodies in combination with venous thromboses. Since PF4-PAA antibodies remained negative, this might have been the reason for not developing thrombocytopenia.

\section{Recent Guidelines and Recommendations to Diagnose and Treat VITT}

Following the recognition of VITT, multiple guidelines have been published to risk stratify patients presenting with possible symptoms after ChAdOx1nCoV-19 vaccinations. ${ }^{10-14}$

These guidelines have been developed based upon the laboratory results and clinical symptoms observed in the initial cases of VITT. Definite cases typically present with a combination of the following symptoms:

- Occurrence within 5 to 28 days after a first vaccination with ChAdOx1 nCoV-19.

- Decrease in platelet count.

- Raised D-dimer.

- Low fibrinogen.

- Positive PF4-induced antibodies (either measured by a PF4-ELISA-assay and/or a PF4-enhanced platelet activation assay).

- Rapid HIT assays like chemiluminescence assays or lateral flow technologies remain negative. ${ }^{15}$

- Acute venous and/or arterial thrombosis.

However, it is becoming increasingly clear that VITT may encompass a broader range of clinicopathological presentations ${ }^{10}$ as observed in the patients presented in this letter to the editor. Recommended treatment options are the avoidance of all types of heparins, the use of intravenous direct thrombin inhibitors, subcutaneous fondaparinux or oral anticoagulation with direct oral anticoagulants, and the immediate application of intravenous immunoglobulins in severe cases to reduce the high mortality rates. In livethreatening situations, additional plasma exchange might be considered. ${ }^{11,12,14,16,17}$

\section{Conclusion}

Our findings have implications for clinical practice: one should be aware that patients can present with diverse clinical symptoms ranging from isolated thrombocytopenia or single-site thrombosis to the full-blown picture of VITT with multiple arterial and/or venous thrombosis and 
thrombocytopenia. The possibility that early detection and therapeutic-dose anticoagulation could prevent thrombosis (as seen in patient 4 ) should be considered; moreover-based on this case series-mRNA vaccines may be safe for use in VITT patients.

\section{Conflict of Interest}

E.L.-L. has received lecture honoraria and advisory fees from Bayer AG, Boehringer Ingelheim, Bristol-Myers Squibb/Pfizer, Daiichi-Sankyo, Portola, CSL Behring, and Aspen, and institutional research support from Bayer AG, Bristol-Myers Squibb/Pfizer, Daiichi-Sankyo, and CSLBehring. L.S. was supported within the Gerhard-Domagk Research Program by the University Medicine Greifswald. M.P. has no conflicts of interest. J.H. has received lecture honoraria and consulting fees from Pfizer, Leo Pharma, Bayer, and Bristol-Myers Squibb. A.G. has received lecture honoraria and advisory fees from Bayer AG, Boehringer Ingelheim, Bristol-Myers Squibb/Pfizer, Daiichi-Sankyo, and Aspen. J.-A.S. has no conflicts of interest. T.E.W. has received lecture honoraria from Alexion and Instrumentation Laboratory, and royalties from Informa (Taylor \& Francis); has provided consulting services to Aspen Canada, Aspen Global, Bayer, CSL Behring, Ergomed, and Octapharma; has received research funding from Instrumentation Laboratory; and has provided expert witness testimony relating to heparin-induced thrombocytopenia (HIT) and non-HIT thrombocytopenic and coagulopathic disorders.

\section{References}

1 Greinacher A, Thiele T, Warkentin TE, Weisser K, Kyrle PA, Eichinger S. Thrombotic thrombocytopenia after ChAdOx1 nCov-19 vaccination. N Engl J Med 2021;384(22):2092-2101

2 Schultz NH, Sørvoll IH, Michelsen AE, et al. Thrombosis and thrombocytopenia after ChAdOx1 nCoV-19 vaccination. N Engl J Med 2021;384(22):2124-2130

3 Pavord S, Scully M, Hunt BJ, et al. Clinical features of vaccineinduced immune thrombocytopenia and thrombosis. N Engl J Med 2021;385(18):1680-1689

4 Gabarin N, Patterson S, Pai M, et al. Venous thromboembolism and mild thrombocytopenia after ChAdOx1 nCoV-19 vaccination. Thromb Haemost 2021;121(12):1677-1680
5 Salih F, Schönborn L, Kohler S, et al. Vaccine-induced thrombocytopenia with severe headache. N Engl J Med 2021;385(22): 2103-2105

6 Kennedy VE, Wong CC, Hong JM, et al. VITT following Ad26.COV2.S vaccination presenting without radiographically demonstrable thrombosis. Blood Adv 2021;5(22):4662-4665

7 Schönborn L, Thiele T, Kaderali L, Greinacher A. Decline in pathogenic antibodies over time in VITT. N Engl J Med 2021; 385(19):1815-1816

8 Greinacher A, Selleng K, Palankar R, et al. Insights in ChAdOx1 nCov-19 vaccine-induced immune thrombotic thrombocytopenia (VITT). Blood 2021;138(22):2256-2268

9 Thiele T, Ulm L, Holtfreter S, et al. Frequency of positive anti-PF4/ polyanion antibody tests after COVID-19 vaccination with ChAdOx1 nCoV-19 and BNT162b2. Blood 2021;138(04):299-303

10 Lavin M, Elder PT, O'Keeffe D, et al. Vaccine-induced immune thrombotic thrombocytopenia (VITT) - a novel clinico-pathological entity with heterogeneous clinical presentations. Br J Haematol 2021;195(01):76-84

11 Elalamy I, Gerotziafas G, Alamowitch S, et al; Scientific Reviewer Committee. SARS-CoV-2 vaccine and thrombosis: an expert consensus on vaccine-induced immune thrombotic thrombocytopenia. Thromb Haemost 2021;121(08):982-991

12 Franchini M, Liumbruno GM, Pezzo M. COVID-19 vaccine-associated immune thrombosis and thrombocytopenia (VITT): diagnostic and therapeutic recommendations for a new syndrome. Eur J Haematol 2021;107(02):173-180

13 Pavord S, Hunt BJ, Horner D, Bewley S, Karpusheff JGuideline Committee. Vaccine induced immune thrombocytopenia and thrombosis: summary of NICE guidance. BMJ 2021;375(2195): n2195

14 Nazy I, Sachs UJ, Arnold DM, et al. Recommendations for the clinical and laboratory diagnosis of VITT against COVID-19: communication from the ISTH SSC Subcommittee on Platelet Immunology. J Thromb Haemost 2021;19(06):1585-1588

15 Sachs UJ, Cooper N, Czwalinna A, et al. PF4-dependent immunoassays in patients with vaccine-induced immune thrombotic thrombocytopenia: results of an interlaboratory comparison. Thromb Haemost 2021;121(12):1622-1627

16 Greinacher A, Langer F, Makris M, et al. Vaccine-induced immune thrombotic thrombocytopenia (VITT): update on diagnosis and management considering different resources. J Thromb Haemost 2021. Doi: $10.1111 /$ jth. 15572

17 Graf T, Thiele T, Klingebiel R, Greinacher A, Schäbitz WR, Greeve I. Immediate high-dose intravenous immunoglobulins followed by direct thrombin-inhibitor treatment is crucial for survival in SarsCovid-19-adenoviral vector vaccine-induced immune thrombotic thrombocytopenia VITT with cerebral sinus venous and portal vein thrombosis. J Neurol 2021;268(12):4483-4485 